\title{
Stability of the Elbow Joint: Relevant Anatomy and Clinical Implications of In Vitro Biomechanical Studies
}

\author{
J. de $\operatorname{Haan}^{1}$, N.W.L. Schep ${ }^{2}$, D. Eygendaal ${ }^{3}$, G-J. Kleinrensink ${ }^{4}$, W.E. Tuinebreijer ${ }^{2}$ and D. den $\operatorname{Hartog}^{*}, 2$ \\ ${ }^{I}$ Department of Surgery-Traumatology, Westfriesgasthuis, P.O. Box 600, 1620 AR Hoorn, The Netherlands \\ ${ }^{2}$ Department of Surgery-Traumatology, Erasmus MC, University Medical Center Rotterdam, P.O. Box 2040, 3000 CA \\ Rotterdam, The Netherlands \\ ${ }^{3}$ Department of Orthopaedics, Amphia Hospital, Molengracht 21, 4818 CK Breda, The Netherlands \\ ${ }^{4}$ Department of Neuroscience, Erasmus MC, University Medical Center Rotterdam, P.O. Box 2040, 3000 CA Rotterdam, \\ The Netherlands
}

\begin{abstract}
The aim of this literature review is to describe the clinical anatomy of the elbow joint based on information from in vitro biomechanical studies. The clinical consequences of this literature review are described and recommendations are given for the treatment of elbow joint dislocation.

The PubMed and EMBASE electronic databases and the Cochrane Central Register of Controlled Trials were searched. Studies were eligible for inclusion if they included observations of the anatomy and biomechanics of the elbow joint in human anatomic specimens.

Numerous studies of the kinematics, kinesiology and anatomy of the elbow joint in human anatomic specimens yielded important and interesting implications for trauma and orthopaedic surgeons.
\end{abstract}

Keywords: Elbow joint, joint instability, anatomy, biomechanics, review literature.

\section{INTRODUCTION}

The elbow joint is the second most commonly dislocated joint in adults [1]. During a period of twelve years, Josefsson et al. reported 178 elbow dislocations (simple and complex) in a population of approximately 243,000 persons, making the annual incidence of elbow dislocations in children and adults 6.1 per 100,000 [2].

Elbow dislocations are classified as simple or complex [3]. Simple dislocations are dislocations without fractures. Complex dislocations are associated with fractures of the distal humerus, radial head, proximal ulna and/or coronoid process. The combination of an elbow dislocation with a fracture of the radial head and the coronoid process is called a "terrible triad" due to the high rate of complications. Surgeons treating elbow dislocation are concerned about two complications: stiffness and instability of the elbow joint. Stiffness or restricted range of motion and instability can be seen as contrasts on a seesaw (Fig. 1). Range of motion can be measured reliably with a standard goniometer for assessing stiffness [4]. Instability can be classified, according to the timing, as acute or chronic instability or recurrent dislocation. In a review of simple elbow dislocation, including eight studies with a total of 342 patients, only one recurrent dislocation was mentioned [5]. Despite this low recurrence rate, surgeons prefer long immobilisation to

*Address correspondence to this author at the Department of SurgeryTraumatology, Erasmus MC, University Medical Center Rotterdam, P.O. Box 2040, 3000 CA Rotterdam, The Netherlands; Tel: +31 10 7032395; Fax: +31 10 7032396; E-mail: d.denhartog@erasmusmc.nl prevent instability over early functional treatment to prevent restricted range of motion [6]. This preference is valid because it is easier to treat stiffness than instability of the elbow joint. For most activities of daily living, restricted range of motion is not a major problem. In a study by Morrey et al., most of the activities of daily living could be accomplished with a $100^{\circ}$ arc of motion (from $30^{\circ}$ to $130^{\circ}$ ) and $100^{\circ}$ of forearm rotation $\left(50^{\circ}\right.$ of pronation and $50^{\circ}$ of supination) [7].

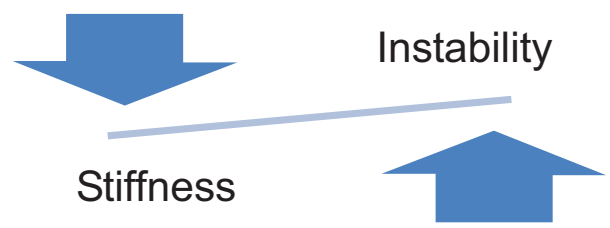

Fig. (1). The two contrasting complications of elbow joint dislocations: stiffness and instability.

During dislocation of the elbow joint, ligamentous damage occurs in a circle from lateral to medial, as described by O'Driscoll [8]. Shortly after dislocation, the elbow joint is unstable when examined during general anaesthesia. In three studies analysing patients with simple elbow dislocations, all $(n=123)$ were evaluated and classified as unstable for valgus stress, when compared to the uninjured side examined under anaesthesia [9-11]. In two of these studies, varus stress was evaluated as well and was present in 39\% (24/61) of all patients. Posterolateral rotatory stability was not assessed in any of these studies $[9,11]$. The operated cases in these studies $(n=78)$ were all explored at the medial side of the elbow. The medial collateral ligament was completely ruptured or avulsed from the 
epicondyle in all cases. In 46 of the 78 operated cases (59\%), the lateral side was explored, and in all cases, the lateral collateral ligament was completely ruptured or avulsed from the epicondyle. Despite these ruptures of both collateral ligaments, patients can be treated functionally, which is characterised by early active motion within the limits of pain with or without the use of a sling, hinged brace or functional plaster. This functional treatment is possible because of the functional, dynamic joint stability. Dynamic joint stability is due to compression forces produced by the muscles crossing the elbow joint. Physical examination of the elbow joint during circumstances that eliminate this dynamic stability can reveal chronic instability long after dislocation of an elbow. For instance, in 24 of the 41 examined cases (59\%), Eygendaal et al. reported persistent medial instability on dynamic radiographs with valgus loads after an average of 9 years after a simple dislocation. Magnetic resonance imaging combined with arthrography revealed that $42 \%$ ( $n=19$ tested) of these cases with medial instability on dynamic radiographs had a rupture of the medial collateral ligament. No recurrent elbow dislocations were noted [12].

The stability and biomechanics of the elbow joint have been examined in numerous studies of human anatomic specimens $[13,14]$. Joint stability can be functionally divided in static and dynamic aspects. Static stability is controlled by the osteoarticular architecture and the capsule and ligamentous parts. Dynamic stability is determined by neuromuscular factors. In the elbow joint, this specifically means that the static part is mainly provided by the congruency between the articulating surfaces at the elbow joint. The other static stabilisers are the anterior joint capsule, the medial and lateral collateral ligaments and the interosseous membrane. The dynamic part includes the muscles that cross the elbow joint. The stabilisers of the elbow joint are divided into primary and secondary constraints (Fig. 2). A primary constraint is defined as a constraint where release causes laxity, and a secondary constraint is a constraint where release alone is insufficient to cause laxity, but where release after division of the fist constraint increases the laxity of the joint. The primary constraints are the anterior medial collateral ligament (AMCL), the lateral collateral ligament complex (LCLC) and the ulnohumeral articulation. The secondary constraints are the radiohumeral articulation, the common flexor-pronator tendon, the common extensor tendon and the capsule.

Stability studies on elbow joints of anatomic specimens often start with an extensive anatomical examination of the collateral ligament complexes. These in vitro biomechanical studies also have important consequences for diagnosis, treatment and rehabilitation of (post)-traumatic injuries of the elbow joint. The aim of this literature review was to describe the clinical anatomy of the elbow joint. The clinical consequences of this literature review are described and recommendations are given for the treatment of dislocation of the elbow joint.

\section{MATERIALS AND METHODS}

The authors have systematically screened the PubMed (until September 2010), EMBASE (1980 - September 2010) and Cochrane Controlled Trials Register electronic databases. As main keywords, we used 'Elbow; Adult; Elbow Joint/in (Injuries); Elbow Joint/su (Surgery); English Abstract; Dislocation; Fractures/su (Surgery); Human; Male; Female; Radius; Radial head; Radius Fractures; Radius Fractures/su (Surgery); Radius Fractures/tr (Treatment).' Articles in languages other than English, French, German or Dutch were excluded. Of all the articles selected, the reference lists were searched for additional articles, surgical reconstruction techniques and postoperative treatments. Studies were eligible for inclusion if they included anatomy and biomechanics of the elbow joint, as observed on human anatomic specimens.

\section{RESULTS}

\section{Anatomy of Ligamentous Elbow Joint Stability}

The literature search retrieved 108 studies. Thirty-nine studies examining biomechanics and anatomy of the elbow joint on human anatomic specimen were selected for this review. Studies about biomechanics of the elbow joint often refer to the article by Schwab et al., published in 1980 [15].

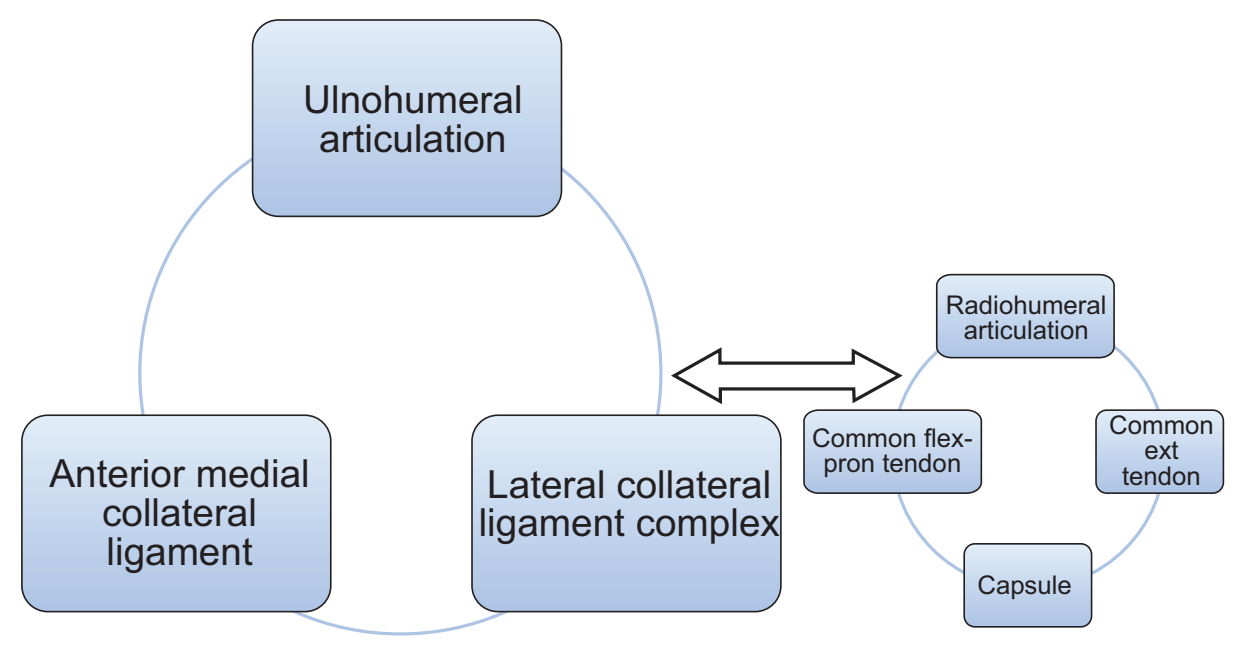

Fig. (2). Primary and secondary constraints to elbow joint stability. 
This study describes the anatomy of the elbow joint and biomechanics of the medial collateral ligament from the clinical point of view. Many of the author's insights were later proven to be true in studies on elbow joints of human anatomic specimens. The humeral origin of the medial collateral ligament (MCL) is reported to be located eccentrically with respect to the axis of rotation of the joint. The MCL consists of an anterior bundle, AMCL, posterior bundle or PMCL and a transverse ligament; the last structure does not span the joint and therefore does not contribute to stability. The AMCL is divided in two functional components and is taut throughout the full range of flexion and extension because the components are alternatively tightening throughout this range of motion. The posterior part of the AMCL is taut from $80^{\circ}$ flexion to full flexion; in contrast, the anterior part of the AMCL is taut in extension. The AMCL is a stronger ligament than the PMCL and acts as the major medial ligamentous joint stabiliser. According to Schwab's article, the lateral collateral ligament (LCL) runs from the lateral epicondyle to the annular ligament (AL) without attachments to the ulna, so the lateral ulnar collateral ligament (LUCL) and the accessory lateral ligament were not described. Morrey and An studied the functional anatomy of the ligaments of the elbow in ten fresh-frozen upper extremities and a quantitative dissection and described the collateral ligaments (Fig. 3) [16]. They describe an MCL complex (MCLC) consisting of the separately defined anterior (oblique) bundle (AMCL), a posterior bundle (PMCL) and a transverse segment. The transverse segment runs from the coronoid to the tip of the olecranon, i.e., one part of the ulna to another part of the ulna. It is often not well defined and apparently contributes little to nothing to elbow stability because it originates from and inserts on the ulna. The transverse part of the MCL is also called Cooper's
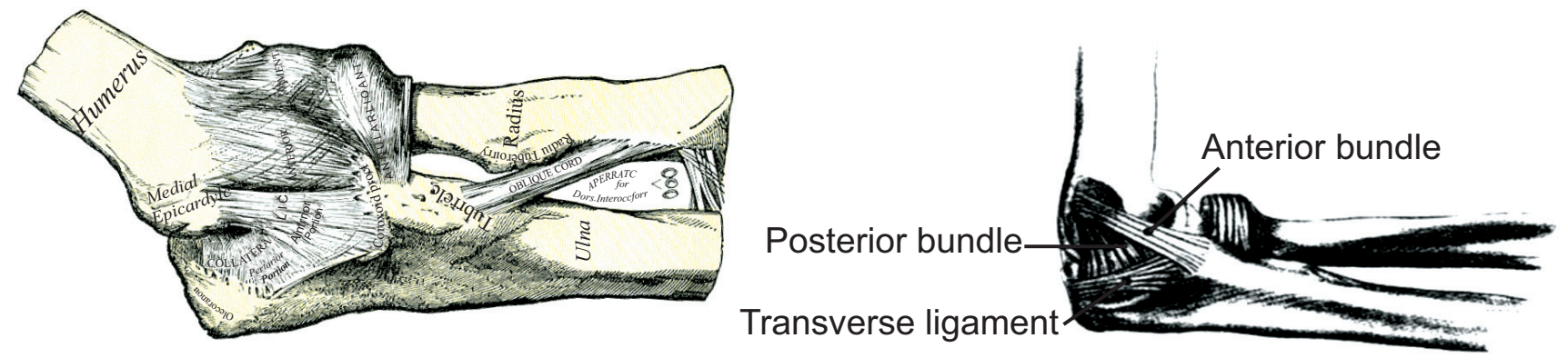

Fig. (3). Left elbow joint with medial collateral ligaments [20].

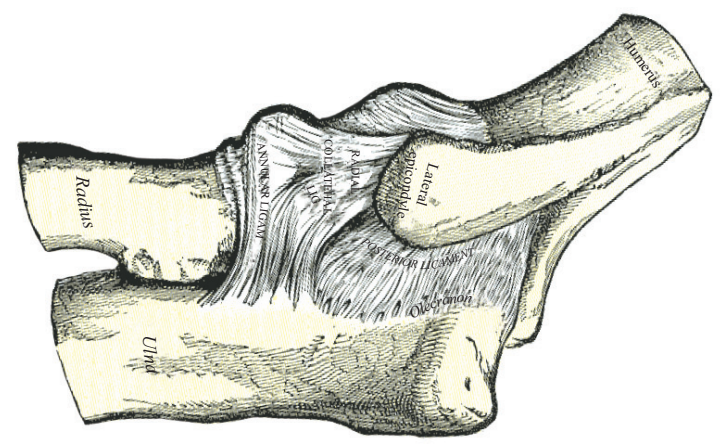

Fig. (4). Left elbow joint with lateral collateral ligaments [20]. ligament [17]. The LCLC consists of the AL, radial collateral ligament or MCL, accessory lateral ligament and the LUCL (Fig. 4). The LCL is poorly demarcated and runs from the lateral epicondyle to the annular ligament. The accessory posterior ligament runs from the lateral epicondyle to the crista musculi supinatoris ulnae of the ulna together with the inferior margin of the AL. In five of the ten specimens, the LUCL runs from the lateral epicondyle to the crista $\mathrm{m}$. supinatoris of the ulna. In a later publication, this ratio of 5 to 10 was corrected: it was found in nine of the specimens and, in the tenth, was present but underdeveloped [18]. In this later anatomical study, the LUCL was observed in all 17 examined fresh-frozen elbows [18]. This LUCL was already described in 1958 by Martin as a bundle, but without naming it: "a definite bundle which normally crosses the annular band and gains attachment to the supinator crest, frequently to a special tubercle on that crest" [19].

Regan et al. [21] extended the functional anatomy study of Morrey and An [16], which defined the axis of rotation of the LCL and MCL and the elongation-tension relationships of the AMCL, PMCL and the LCL. In the first part of this study, the ranges of the elbow joint angle were examined. In this area, the ligaments of the elbow are tense or taut. The AMCL and LCL were taut throughout almost the entire range of flexion. The PMCL was taut only when the elbow was in a flexed position. In the second part of this study, the structural properties of each collateral ligament were determined. The load to failure of each ligament was studied. The AMCL was the strongest and stiffest ligament, followed by the LCL. The weakest ligament was the PMCL.

Cohen et al. examined 40 elbows of human anatomic specimens to characterise the anatomy of the LCLC [22]. The LCL and the AL formed a broad conjoined insertion

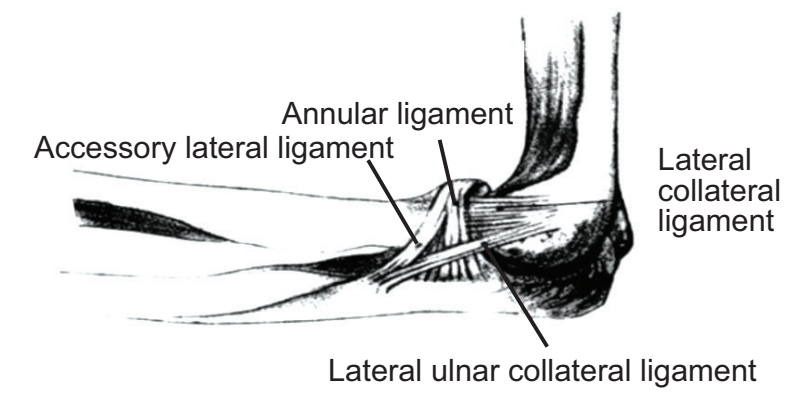

Lateral ulnar collateral ligament 

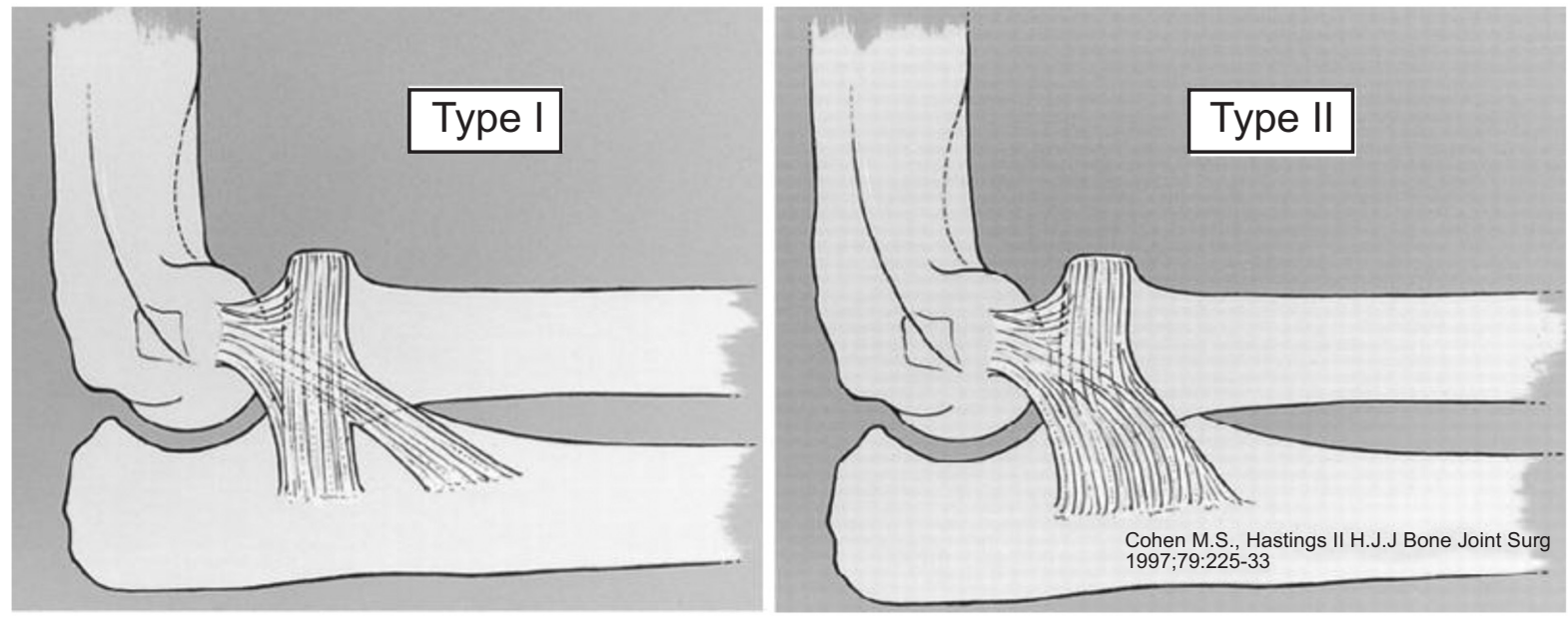

Fig. (5). Insertion (type 1 and 2) of the lateral collateral ligament and annular ligament onto the ulna. *Reprinted from Journal of Bone and Joint Surgery American, 1997, volume 79, 2, Rotatory instability of the elbow. The anatomy and role of the lateral stabilizers, Cohen MS and Hastings H, 225-233, with permission from Rockwater and Journal of Bone and Joint Surgery.

onto the proximal aspect of the ulna in all 40 specimens (Fig. 5). This conjoined tendon became taut with the forearm upon supination. In 22 specimens, the LCLC had a double, bidirectional insertion onto the ulna. In 18 specimens, a single broad conjoined ligament inserted onto the ulna. A separate band from the lateral epicondyle to the ulna, such as the LUCL in Fig. (3), was not identified.

The LCL and AL became confluent with the overlying supinator tendon and so the supinator tendon reinforced the LCL and AL. A distinct band of the extensor carpi ulnaris fascia coursed from the inferior aspect of the lateral epicondyle to the ulna in 36 specimens.

Another anatomical study was performed by Beckett et al., who examined the anatomical variations of the medial and lateral collateral ligament complexes [23]. These variations occurred on the medial side in $50 \%$ of the 39 cadaveric elbow joints and on the lateral side in $25 \%$. The MCLC and LCLC were divided in four distinct groups.

Group MCLC 1 (49\%) was the normal group with classic AMCL, PMCL and transverse or oblique band anatomy.

Group MCLC 2 was the strong oblique group (28\%), with a broad fan-shaped insertion of the transverse or oblique band not only at the coronoid process but also in the anterior band.

Group MCLC $3(8 \%)$ resembled the normal group but had an additional band passing from the posterior capsule to the oblique or transverse band.

Group MCLC $4(15 \%)$ exhibited a pattern that was a combination of that exhibited in groups 2 and 3 .

In group LCLC $1(23 \%)$, only the LCL and AL were present.

In group LCLC $2(44 \%)$, the LCL, AL and LUCL were present.

Group LCLC 3 (25\%) exhibited the LCL, the AL and an accessory collateral ligament.

Group LCLC 4 (7\%) exhibited a pattern that was a combination of that exhibited in groups 2 and 3 .
A remarkable result of this study is that the transverse or oblique band of the MCLC is not poorly developed, as reported earlier [16]. The accessory collateral ligament of the LCLC was already described in the study by Martin and the study by Morrey and An as the "accessory posterior ligament" [16, 19].

Callaway et al. examined the anatomy of the anterior bundle of the medial collateral ligament (AMCL) in 28 anatomic specimens [24]. The AMCL consisted of anterior and posterior bands that tightened in reciprocal fashion as the elbow was flexed and extended. The anterior band of the AMCL is taut in extension and relaxed in flexion; the posterior band of the AMCL behaves in a reciprocal fashion. Isometric fibres are found between the anterior and posterior bands of the AMCL (Fig. 6).

Fuss also examined the MCL by visual inspection for the presence of taut fibre bundles [17]. He also concluded that the posterior part of the MCL was taut when the elbow was fully flexed. The anterior part of the MCL contained some fibres that were taut in full extension and some that were taut in any position (isometric fibres). Because this bundle guides the joint's movements, Fuss called these fibres "the guiding bundle."

However, Armstrong et al. could not find true isometric fibres in the AMCL during combined supination and passive flexion in twelve elbow specimens [25]. The smallest distance between the attachment sites of the AMCL on the medial epicondyle and the ulna was found on the lateral aspect of the attachment site of the AMCL on the medial epicondyle. This point was near the axis of rotation.

An anatomic and histological study of the LCLC was performed by Imatani et al. [26]. Fifteen elbow specimens were examined to characterise macroscopic and microscopic aspects of the LCLC. The LUCL adhered closely to the supinator and extensor muscles, its intermuscular fascia and the anconeus muscle. The LUCL lies posterior to the LCL and the extensor carpi ulnaris muscle. Microscopically, the LUCL was a slender and vague structure and consisted of the thick area of the capsuloligamentous layer. 


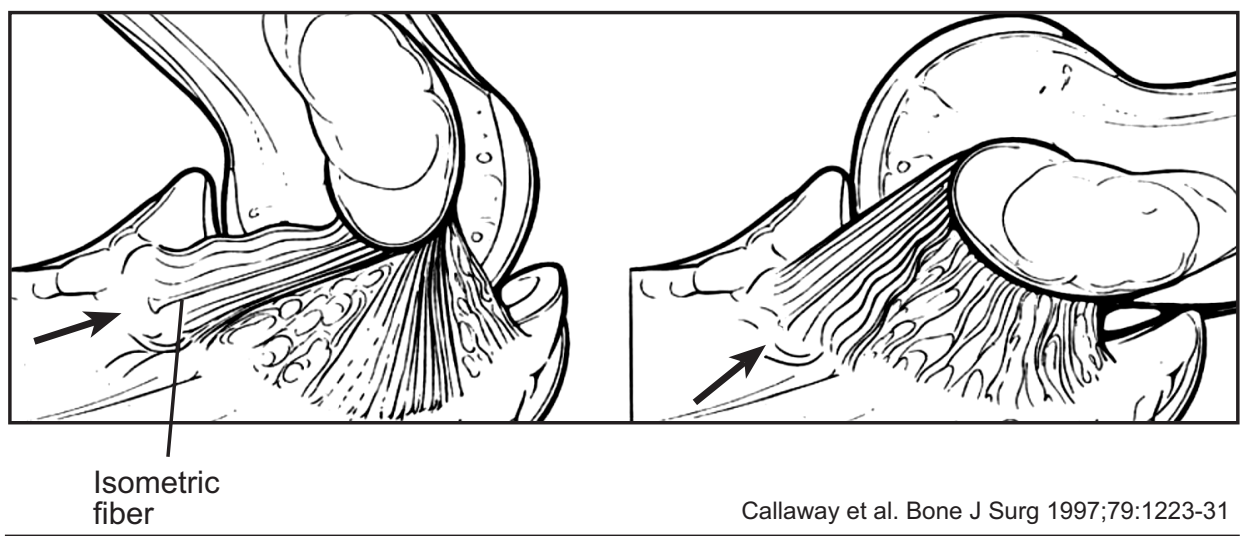

Fig. (6). The anterior and posterior bands of the anterior bundle (AMCL) tighten in reciprocal fashion as the elbow is flexed and extended; they are separated by isometric fibres (arrows). Reprinted from Journal of Bone and Joint Surgery American, 1997, volume 79, 8, Biomechanical evaluation of the medial collateral ligament of the elbow, Callaway GH, Field LD, Deng XH, et al. 1223-1231, with permission from Rockwater and Journal of Bone and Joint Surgery.

Floris et al. examined the anatomy of the AMCL in 18 osteoligamentous elbow joint specimens [27]. In all specimens, the AMCL was a distinct structure with a macroscopically visible ridge between the anterior and posterior bands.

Seki et al. published a study of the LCLC in five osteoligamentous elbow preparations, which was a continuation of a previous study written in Japanese [28]. This Japanese study revealed that the LCLC of the elbow has a Y-shaped configuration, which consists of a superior, an anterior and a posterior band (Fig. 7).

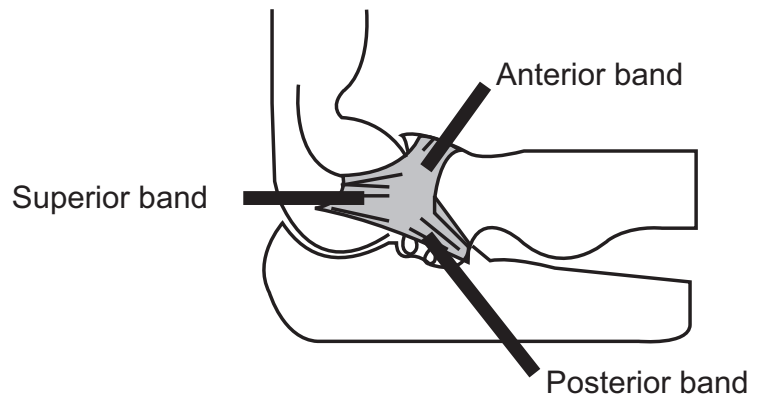

Seki et al. J Shoulder Elbow Surg 2002;11:53-9

Fig. (7). The three bands of the Y structure in the lateral collateral ligament complex (LCLC). Reprinted from Journal of Shoulder and Elbow Surgery, volume 11, Seki A, Olsen BS, Jensen SL, Eygendaal D, Sojbjerg JO, Functional anatomy of the lateral collateral ligament complex of the elbow: configuration of $\mathrm{Y}$ and its role, 53-59, Copyright 2002, with permission from Elsevier.

Regan and Morrey examined 35 patients with a coronoid fracture of the ulna and developed a classification system for coronoid fractures: type I with avulsion of the tip, type II with a fragment involving $50 \%$ or less of the process, and type III with a fragment involving more than $50 \%$ of the coronoid process [29]. This classification correlated well with the clinical outcome.

Cage et al. investigated 20 elbows of human anatomic specimens to identify the soft tissue attachments of the coronoid process and correlated this anatomy with the radiographic classification of Regan and Morrey [30]. The AMCL insertion had an average distance of $18.4 \mathrm{~mm}$ dorsal to the coronoid tip and was attached to the free bone fragment, only in type III fractures. The capsule inserted an average distance of $6.4 \mathrm{~mm}$ distal to the coronoid tip; in only three of the 20 specimens, the capsule inserted to the tip of the coronoid. Type I fractures are usually intra-articular and the anterior capsule is attached to the fragment of type II fractures. The brachialis muscle had insertions at the elbow capsule, coronoid and proximal ulna. The bony insertion had an average length of $26.3 \mathrm{~mm}$, with its proximal margin an average distance of $11 \mathrm{~mm}$ to the coronoid tip. Only type III fractures are large enough to include the brachialis tendon insertion and the AMCL insertion.

Takigawa et al. have investigated the anatomy and function of the LUCL in 26 fresh-frozen anatomic specimens [31]. The LCLC consisted of three main fibre bundles. The LCL ran from the inferior part of the lateral epicondyle and blended with the AL. The AL ran around the radial head and was confluent with the LCL and the LUCL. The LUCL was found in all 26 specimens and was thin and slender. The LUCL ran from the inferior part of the lateral epicondyle and blended with the AL. The distal part of the LUCL, which ran from the AL to the supinator crest of the ulna, had three configurations. The first two types were the same as type I $(n=8$, bilobed) and the broad conjoined type II $(n=9)$ from the study by Cohen and Hastings [22]. Takigawa and coworkers also described a third type in nine specimens in which the lateral ligaments inserted to the ulnar supinator crest in a broad single expansion, accompanied by a thin membranous fibre between the proximal and distal fibres.

\section{Load Transfer to the Elbow Joint}

In a study on seven upper limbs of anatomic specimens, the static axial load to the hand with the elbow extended was transferred to the radiohumeral joint among $57 \%$ of patients and to the ulnohumeral joint among 43\% [32]. Morrey and co-workers observed the highest load on the radiohumeral joint with the forearm in pronation and between zero and $30^{\circ}$ of flexion [33]. Flexion and pronation increase the contact between the radial head and capitellum. With the elbow in valgus alignment, defined as contact between the radial head 
and the capitellum, the load was transferred to the ulnohumeral joint in 3\% of patients [34]. In varus alignment of the elbow, defined as no contact between the radial head and capitellum, the load was transferred to the ulnohumeral joint in $93 \%$ of patients. Therefore, load transfer is dependent on the rotational position of the forearm, the amount of flexion, and the varus or valgus position of the elbow.

\section{The Role of Muscle Load in Providing Elbow Joint Stability}

Dunning et al. determined the contribution of muscle force and forearm position to the stability of the LCLC-deficient elbow in ten fresh-frozen upper extremities, using a testing system that was capable of simulating active motion [35]. Simulated motion revealed less variability in measurements in comparison with manual passive motion [36, 37]. The distal tendons of three elbow flexors (biceps, brachialis and brachioradialis), the principal extensor (triceps) and the pronator teres were connected to steel cables and could be selectively and sequentially loaded to generate the desired motion. The LCL was divided at its insertion at the lateral epicondyle. At each angle of elbow flexion, the varus and valgus laxity of the ulna relative to the humerus were calculated. The extremity was examined in the following positions: vertical, varus and valgus gravity orientations. Gross instability was present after LCL transection during passive elbow flexion, with the arm in the varus orientation. With the arm in vertical orientation and the forearm in supination, sectioning of the LCL increased rotatory instability compared to values obtained with the forearm in pronation and with the intact LCL. Therefore forearm pronation stabilised the LCL-deficient elbow during passive flexion with the arm in the vertical orientation. This rotatory instability with the forearm in supination was reduced significantly when active flexion was simulated. The authors suggest that, in patients with acute posterolateral rotatory instability after elbow dislocations and extended lateral surgical exposures, passive elbow flexion with the forearm in pronation can be used for rehabilitation. Furthermore, splinting of these elbows should be performed using a brace, with the forearm held in full pronation. Armstrong et al. repeated the former study in ten cadaveric upper extremities, but instead of the LCL, the MCL (AMCL and PMCL) was sectioned to generate valgus instability [38]. Following transection of the MCL, the elbow was more stable in supination than pronation during passive flexion. Rotatory instability with the forearm pronated was reduced significantly when active flexion was simulated. The authors advise active motion for the MCL-deficient elbow during the early stages of healing because active motion provides stability similar to that of an intact joint. In contrast, passive motion may cause insignificant stability, particularly with the forearm maintained in pronation. Therefore splinting and passive mobilisation for the MCL-deficient elbow should be done with the forearm in supination.

\section{Kinematics of the Elbow Joint}

The elbow is a hinge joint allowing flexion and extension. The proximal radioulnar joint permits rotation, which involves both pronation and supination. According to guidelines proposed by The American Academy of Orthopaedic Surgeons, which are based on four sources, the average range of elbow motion is: flexion $146^{\circ}$, extension $0^{\circ}$, pronation $71^{\circ}$ and supination $84^{\circ}$ [39].

In a study with five fresh frozen upper extremities, maximum of $7.6^{\circ}$ was observed in the valgus-varus direction of the ulnohumeral joint during flexion, with the weight of the forearm as stress [40]. Maximal internal and external rotation of the ulnohumeral joint $\left(5.3^{\circ}\right)$ was observed during flexion of the elbow joint.

\section{Clinical Significance of In Vitro Biomechanical Studies of the Elbow Joint}

When interpreting biomechanical studies, it is important to distinguish between studies with or without simulation of active muscle loading. It is important that the testing apparatus is able to measure the dynamic muscular aspect of joint stability. The stability provided by muscular loading across the elbow joint produced less variable measurements $[36,37]$.

\section{Stability Testing of the Elbow Joint}

Stability testing should be performed in positions such that the collateral ligaments are lax.

The optimal positions for valgus testing in different studies were diverse. Therefore, a final conclusion with regard to the best position is not possible; the positions with the greatest laxity were: $90^{\circ}$ of flexion [41], $60-70^{\circ}$ of flexion [42], in pronation to compensate for the deficient lateral structures (if stable in pronation, the AMCL can be assumed to be intact) [8], $90^{\circ}$ of flexion with respect to the AMCL [24], pronation or neutral forearm rotation [43], AMCL at $30-40^{\circ}$ and entire MCL at $80-100^{\circ}$ of flexion [27], $70-90^{\circ}$ of flexion, and forearm in pronation or neutral forearm rotation (to prevent radial head luxation in cases of additional LCLC lesions) [44].

The results for the pivot shift test according to different studies were: supination of the forearm; application of valgus stress and an axial compression force to the elbow while it was flexed from full extension [45]; neutral or varus stress in a semi-flexed position $\left(10-30^{\circ}\right)$ of the elbow and supination of the forearm [46].

Positions for varus testing were described in two studies. In the first study, the flexion angle was not a significant factor [41]. In the second study, the maximum joint laxity in forced varus and external rotation (supination) occurred between $90-110^{\circ}$ of flexion [47].

\section{Reducing Elbow Dislocations and Related Postoperative Treatment}

O'Driscoll et al. recommended that posterior elbow dislocations should be reduced in supination [8]. After reducing the dislocation, the elbow should be tested for valgus stability in pronation. If the elbow is stable in pronation, the AMCL can be assumed to be intact and the elbow can be treated immediately with a hinged cast-brace, with the forearm in full pronation. Jensen et al. concluded that supination provided the greatest stability during postoperative treatment for anteromedial dislocations; neutral rotation provided the greatest stability for posteromedial dislocation; pronation of the forearm was most effective for posterolateral dislocations [48]. Dunning 
et al. performed a study with simulated active motion and found that in patients with acute posterolateral rotatory instability after elbow dislocations and in patients with extended lateral surgical exposures, passive elbow flexion with the forearm in pronation can be used in rehabilitation because forearm pronation stabilised the LCL-deficient elbow [35]. Splinting of these elbows should be done using a brace with the forearm held in full pronation. Instability of the LCL-deficient elbow was not observed during simulated active flexion. It can be concluded that muscle activity is an important posterolateral stabiliser of the elbow; strengthening of these muscles might reduce symptoms of chronic posterolateral rotatory instability.

A very large number of activities of daily living occur with the upper extremity in a shoulder-abducted position and produce varus moments at the elbow joint. This is the reason that the LCLC of the elbow joint should be protected from varus stress after a dislocation by keeping the forearm pronated.

\section{Postoperative Treatment for MCL Repair}

Immobilisation, when deemed necessary after repair of the MCL, should be performed with some degree of flexion. The anterior and posterior bands of the AMCL tighten in reciprocal fashion during flexion and extension of the elbow. The anterior band/part of the AMCL is the most important band and flexion may relax this important band. Dunning et al. advised active motion for the MCL elbow during the early stages of healing because active motion provides stability similar to that of an intact joint [38]. In contrast, passive motion may cause significant stability, particularly with the forearm maintained in pronation. Consequently, splinting and passive mobilisation for the MCL-deficient elbow should be performed with the forearm in supination.

\section{Surgical Reconstructions}

Nielsen and Olsen observed no influence of capsule puncture or transection of the anterior and/or posterior capsule on joint laxity in any studied direction or on the pivot-shift test [49]. Therefore, the authors advise against closure of the joint capsule after elbow surgery in elbows with intact collateral ligaments to prevent capsular contractures.

When performing a surgical procedure at the lateral side of the elbow joint, either the LUCL or the LCL should remain intact to prevent posterolateral rotatory instability [50]. Deutch et al. came to the same conclusion: either the anterior or posterior part of the LCLC can be transected without inducing posterolateral rotatory instability [51]. Reconstructing the LUCL, e.g., for posterolateral rotatory instability, should be done between $30-40^{\circ}$ of flexion, which were the initial values for strain in the LUCL [31]. It is not necessary to fully pronate the forearm because maximum strains in the proximal fibres of the LUCL were not influenced by forearm position.

In the case of a ruptured MCL with a fractured radial head, isolated repair of the ligament is superior to the isolated prosthetic replacement and may be sufficient to restore valgus and internal rotatory stability [52]. In the case of a ruptured LCL with a fractured radial head, isolated repair of the ligament is superior to isolated prosthetic replacement and may be sufficient to restore varus and external rotatory stability $[53,54]$.

When treating an isolated coronoid process fracture type I or II, early motion may be allowed, as there appears little risk of posterior subluxation. However, even an isolated coronoid process fracture type III can lead to a posterior subluxation, especially during $60-105^{\circ}$ elbow flexion [55]. Therefore open reduction and internal fixation are preferred for coronoid process fracture type III. Beingessner and coworkers advise repair of coronoid fracture type II and III in combination with LCLC repair [56]. During rehabilitation, the preferred position of the forearm is supination. Schneeberger and co-workers recommend coronoid reconstruction and radial head replacement with a rigid implant for the elbow in cases of coronoid process/radial head fracture [57]. If elbow instability persists after an elbow dislocation combined with a type I coronoid fracture, repair of both collateral ligaments with an intact, repaired or replaced radial head should restore elbow stability [58]. In these cases, suture fixation of type I coronoid fracture had little effect on elbow stability.

\section{Hinged External Fixator}

The study of Stavlas and co-workers involved use of the Orthofix elbow external fixator (Orthofix, Verona, Italy) and showed that the hinged external fixator restored the stability of the unstable elbow joint after division of the LCL and MCL in eight cadaveric elbow joints [59]. Although the range of motion decreased, the extensor and fixator forced the unstable joint toward the varus position, as compared with the intact elbow. The authors suggest that this constraint to extension may result from the constant and fixed flexionextension axis in the external fixator tested. This is in contrast with the physiology of the joint, in which the flexion-extension axis of the elbow joint is not constant during motion.

Another study with a laterally applied hinged external fixator (Dynamic Joint Distractor II, Stryker Howmedica, Rutherford, New Jersey) was performed by Kamineni et al. in the intact elbow and after division of the LCL and/or MCL in six fresh-frozen anatomic specimens of upper extremities [60]. Varus displacements could be prevented with the external fixator. However, valgus displacements occurred during a load of seven Newtons after either sectioning the LCL or MCL and occurred during every load after sectioning both the LCL and MCL. Therefore, patients with a hinged external fixator for elbow instability should be told not to lift more than the weight of a glass of water. However, during the activities of daily living, valgus stress is rare and short, while most functions generate a sustained varus stress.

\section{CONCLUSIONS}

Numerous studies of the kinematics/kinesiology and anatomy of the elbow joint based on human anatomic specimens of elbow joints yielded important and interesting implications for trauma and orthopaedic surgeons.

Testing of instability should be done with lax collateral ligaments, which means flexion (from $10^{\circ}$ to $90^{\circ}$ ) and pronation of the forearm for valgus testing of the MCL and flexion and supination for varus testing. The pivot-shift test 
is performed with the forearm in supination and with application of valgus stress and an axial compression force to the elbow while it is flexed from full extension. Rehabilitation should be performed with a stable joint, which for valgus instability involves supination of the forearm and for varus instability requires pronation. For both instabilities, active mobilisation should be stimulated to improve muscular stability. Reduction of a posterior elbow dislocation should be carried out with a lax LCLC and therefore with a supinated forearm. Mobilisation with a pronated forearm should be performed subsequently. A surgical approach to the lateral side of the elbow should protect either the LUCL or the LCL. Open reduction and internal fixation of coronoid fracture type II and III in combination with LCLC repair is advisable. A laterally applied hinged external fixator protects primarily against varus stress, so valgus stress should be avoided.

\section{AUTHORS' CONTRIBUTIONS}

All authors have made substantial contributions to conception and design of the review and the drafting and revising of the manuscript. All authors have read and approved the final manuscript.

\section{REFERENCES}

[1] Linscheid RL. Elbow dislocations. In: Morrey BF Ed, The elbow and its disorders. Philadelphia, WB Saunders Company 2010; 41432.

[2] Josefsson PO, Nilsson BE. Incidence of elbow dislocation. Acta Orthop Scand 1986; 57(6): 537-8.

[3] Hildebrand KA, Patterson SD, King GJ. Acute elbow dislocations: simple and complex. Orthop Clin North Am 1999; 30(1): 63-79.

[4] Armstrong AD, MacDermid JC, Chinchalkar S, Stevens RS, King GJ. Reliability of range-of-motion measurement in the elbow and forearm. J Shoulder Elbow Surg 1998; 7(6): 573-80.

[5] de Haan J, Schep NW, Tuinebreijer WE, Patka P, den Hartog D. Simple elbow dislocations: a systematic review of the literature. Arch Orthop Trauma Surg 2010; 130(2): 241-9.

[6] de Haan J, Schep NWL, Peters RW, Tuinebreijer WE, den Hartog D. Simple elbow dislocations in the Netherlands: what are Dutch surgeons doing? Netherlands J Traumatol 2009; 17(5): 124-7.

[7] Morrey BF, Askew LJ, Chao EY. A biomechanical study of normal functional elbow motion. J Bone Joint Surg Am 1981; 63(6): 8727.

[8] O'Driscoll SW, Morrey BF, Korinek S, An KN. Elbow subluxation and dislocation. A spectrum of instability. Clin Orthop Relat Res 1992; (280): 186-97.

[9] Josefsson PO, Gentz CF, Johnell O, Wendeberg B. Surgical versus non-surgical treatment of ligamentous injuries following dislocation of the elbow joint. A prospective randomized study. J Bone Joint Surg Am 1987; 69(4): 605-8.

[10] Josefsson PO, Gentz CF, Johnell O, Wendeberg B. Surgical versus nonsurgical treatment of ligamentous injuries following dislocations of the elbow joint. Clin Orthop Relat Res 1987; 214 : 165-9.

[11] Josefsson PO, Johnell O, Wendeberg B. Ligamentous injuries in dislocations of the elbow joint. Clin Orthop Relat Res 1987; 221: 221-5.

[12] Eygendaal D, Verdegaal SH, Obermann WR, van Vugt AB, Pöll RG, Rozing PM. Posterolateral dislocation of the elbow joint. Relationship to medial instability. J Bone Joint Surg Am 2000; 82(4): 555-60.

[13] Bryce CD, Armstrong AD. Anatomy and biomechanics of the elbow. Orthop Clin North Am 2008; 39(2): 141-54.

[14] Lockard M. Clinical biomechanics of the elbow. J Hand Ther 2006; 19(2): 72-80.

[15] Schwab GH, Bennett JB, Woods GW, Tullos HS. Biomechanics of elbow instability: the role of the medial collateral ligament. Clin Orthop Relat Res 1980; 146: 42-52.

[16] Morrey BF, An KN. Functional anatomy of the ligaments of the elbow. Clin Orthop Relat Res 1985; 201: 84-90.
[17] Fuss FK. The ulnar collateral ligament of the human elbow joint. Anatomy, function and biomechanics. J Anat 1991; 175: 203-12.

[18] O'Driscoll SW, Horii E, Morrey BF, Carmichael SW. Anatomy of the ulnar part of the lateral collateral ligament of the elbow. Clin Anat 1992; 5: 296-303.

[19] Martin BF. The annular ligament of the superior radio-ulnar joint. J Anat 1958; 92(3): 473-82.

[20] Gray H. Anatomy of the human body. 20 ed. Philadelphia: Lea \& Febiger; 1918.

[21] Regan WD, Korinek SL, Morrey BF, An KN. Biomechanical study of ligaments around the elbow joint. Clin Orthop Relat Res 1991; 271: 170-9.

[22] Cohen MS, Hastings H. Rotatory instability of the elbow. The anatomy and role of the lateral stabilizers. J Bone Joint Surg Am 1997; 79(2): 225-33.

[23] Beckett KS, McConnell P, Lagopoulos M, Newman RJ. Variations in the normal anatomy of the collateral ligaments of the human elbow joint. J Anat 2000; 197(3): 507-11.

[24] Callaway GH, Field LD, Deng XH, et al. Biomechanical evaluation of the medial collateral ligament of the elbow. J Bone Joint Surg Am 1997; 79(8): 1223-31.

[25] Armstrong AD, Ferreira LM, Dunning CE, Johnson JA, King GJ. The medial collateral ligament of the elbow is not isometric: an in vitro biomechanical study. Am J Sports Med 2004; 32(1): 85-90.

[26] Imatani J, Ogura T, Morito Y, Hashizume H, Inoue H. Anatomic and histologic studies of lateral collateral ligament complex of the elbow joint. J Shoulder Elbow Surg 1999; 8(6): 625-7.

[27] Floris S, Olsen BS, Dalstra M, Sojbjerg JO, Sneppen O. The medial collateral ligament of the elbow joint: anatomy and kinematics. J Shoulder Elbow Surg 1998; 7(4): 345-51.

[28] Seki A, Olsen BS, Jensen SL, Eygendaal D, Sojbjerg JO. Functional anatomy of the lateral collateral ligament complex of the elbow: configuration of Y and its role. J Shoulder Elbow Surg 2002; 11(1): 53-9.

[29] Regan W, Morrey B. Fractures of the coronoid process of the ulna. J Bone Joint Surg Am 1989; 71(9): 1348-54.

[30] Cage DJ, Abrams RA, Callahan JJ, Botte MJ. Soft tissue attachments of the ulnar coronoid process. An anatomic study with radiographic correlation. Clin Orthop Relat Res 1995; 320: 154-8.

[31] Takigawa N, Ryu J, Kish VL, Kinoshita M, Abe M. Functional anatomy of the lateral collateral ligament complex of the elbow: morphology and strain. J Hand Surg Br 2005; 30(2): 143-7.

[32] Halls AA, Travill A. Transmission of pressures acroos the elbow joint. Anat Rec 1964; 150: 243-7.

[33] Morrey BF, An KN, Stormont TJ. Force transmission through the radial head. J Bone Joint Surg Am 1988; 70(2): 250-6.

[34] Markolf KL, Lamey D, Yang S, Meals R, Hotchkiss R. Radioulnar load-sharing in the forearm. A study in cadavera. J Bone Joint Surg Am 1998; 80(6): 879-88.

[35] Dunning CE, Zarzour ZD, Patterson SD, Johnson JA, King GJ. Muscle forces and pronation stabilize the lateral ligament deficient elbow. Clin Orthop Relat Res 2001; 388: 118-24.

[36] Dunning CE, Duck TR, King GJ, Johnson JA. Simulated active control produces repeatable motion pathways of the elbow in an in vitro testing system. J Biomech 2001; 34(8): 1039-48.

[37] Johnson JA, Rath DA, Dunning CE, Roth SE, King GJ. Simulation of elbow and forearm motion in vitro using a load controlled testing apparatus. J Biomech 2000; 33(5): 635-9.

[38] Armstrong AD, Dunning CE, Faber KJ, Duck TR, Johnson JA, King GJ. Rehabilitation of the medial collateral ligament-deficient elbow: an in vitro biomechanical study. J Hand Surg Am 2000; 25(6): 1051-7.

[39] American Academy of Orthopaedic Surgeons. Joint motion: method of measuring and recording. Chicago 1965.

[40] Tanaka S, An K-N, Morrey BF. Kinematics and laxity of ulnohumeral joint under valgus-varus stress. J Musculoskeletal Res 1998; 2(1): 45-54.

[41] Morrey BF, An KN. Articular and ligamentous contributions to the stability of the elbow joint. Am J Sports Med 1983; 11(5): 315-9.

[42] Sojbjerg JO, Ovesen J, Nielsen S. Experimental elbow instability after transection of the medial collateral ligament. Clin Orthop Relat Res 1987; 218 : 186-90.

[43] Olsen BS, Sojbjerg JO, Nielsen KK, Vaesel MT, Dalstra M, Sneppen O. Posterolateral elbow joint instability: the basic kinematics. J Shoulder Elbow Surg 1998; 7(1): 19-29. 
[44] Eygendaal D, Olsen BS, Jensen SL, Seki A, Sojbjerg JO. Kinematics of partial and total ruptures of the medial collateral ligament of the elbow. J Shoulder Elbow Surg 1999; 8(6): 612-6.

[45] O'Driscoll SW, Bell DF, Morrey BF. Posterolateral rotatory instability of the elbow. J Bone Joint Surg Am 1991; 73(3): 440-6.

[46] Deutch SR, Jensen SL, Olsen BS, Sneppen O. Elbow joint stability in relation to forced external rotation: An experimental study of the osseous constraint. J Shoulder Elbow Surg 2003; 12(3): 287-92.

[47] Olsen BS, Sojbjerg JO, Dalstra M, Sneppen O. Kinematics of the lateral ligamentous constraints of the elbow joint. J Shoulder Elbow Surg 1996; 5(5): 333-41.

[48] Jensen SL, Olsen BS, Seki A, Ole SJ, Sneppen O. Radiohumeral stability to forced translation: an experimental analysis of the bony constraint. J Shoulder Elbow Surg 2002; 11(2): 158-65.

[49] Nielsen KK, Olsen BS. No stabilizing effect of the elbow joint capsule. A kinematic study. Acta Orthop Scand 1999; 70(1): 6-8.

[50] Dunning CE, Zarzour ZD, Patterson SD, Johnson JA, King GJ. Ligamentous stabilizers against posterolateral rotatory instability of the elbow. J Bone Joint Surg Am 2001; 83-A(12): 1823-8.

[51] Deutch SR, Olsen BS, Jensen SL, Tyrdal S, Sneppen O. Ligamentous and capsular restraints to experimental posterior elbow joint dislocation. Scand J Med Sci Sports 2003; 13(5): 311-6.

[52] Jensen SL, Deutch SR, Olsen BS, Sojbjerg JO, Sneppen O. Laxity of the elbow after experimental excision of the radial head and division of the medial collateral ligament. Efficacy of ligament repair and radial head prosthetic replacement: a cadaver study. J Bone Joint Surg Br 2003; 85(7): 1006-10.
[53] Deutch SR, Jensen SL, Tyrdal S, Olsen BS, Sneppen O. Elbow joint stability following experimental osteoligamentous injury and reconstruction. J Shoulder Elbow Surg 2003; 12(5): 466-71.

[54] Jensen SL, Olsen BS, Tyrdal S, Sojbjerg JO, Sneppen O. Elbow joint laxity after experimental radial head excision and lateral collateral ligament rupture: efficacy of prosthetic replacement and ligament repair. J Shoulder Elbow Surg 2005; 14(1): 78-84.

[55] Closkey RF, Goode JR, Kirschenbaum D, Cody RP. The role of the coronoid process in elbow stability. A biomechanical analysis of axial loading. J Bone Joint Surg Am 2000; 82-A(12): 1749-53.

[56] Beingessner DM, Dunning CE, Stacpoole RA, Johnson JA, King GJW. The effect of coronoid fractures on elbow kinematics and stability. Clin Biomech 2007; 22(2): 183-90.

[57] Schneeberger AG, Sadowski MM, Jacob HA. Coronoid process and radial head as posterolateral rotatory stabilizers of the elbow. $\mathrm{J}$ Bone Joint Surg Am 2004; 86-A(5): 975-82.

[58] Beingessner DM, Stacpoole RA, Dunning CE, Johnson JA, King GJ. The effect of suture fixation of type I coronoid fractures on the kinematics and stability of the elbow with and without medial collateral ligament repair. J Shoulder Elbow Surg 2007; 16(2): 213-7.

[59] Stavlas P, Jensen SL, Sojbjerg JO. Kinematics of the ligamentous unstable elbow joint after application of a hinged external fixation device: a cadaveric study. J Shoulder Elbow Surg 2007; 16(4): 491-6.

[60] Kamineni S, Hirahara H, Neale P, O'Driscoll SW, An KN, Morrey BF. Effectiveness of the lateral unilateral dynamic external fixator after elbow ligament injury. J Bone Joint Surg Am 2007; 89(8): 1802-9.

(C) de Haan et al.; Licensee Bentham Open.

This is an open access article licensed under the terms of the Creative Commons Attribution Non-Commercial License (http://creativecommons.org/licenses/by-nc/3.0/) which permits unrestricted, non-commercial use, distribution and reproduction in any medium, provided the work is properly cited. 